\title{
Hyperemesis Induced Acute Quadriparesis In Pregnancy - A Case Report.
}

\author{
${ }^{1}$ Dr. Archana Gupta, MD, ${ }^{2}$ Dr. Gaurav kavi, MD, ${ }^{3}$ Dr. Neelima Singh, MD, \\ ${ }^{4}$ Dr. Sankalp Dwivedi, MBBS
}

\begin{abstract}
Reports on quadriparesis in pregnancy secondary to gastrointestinal losses are rare. We describe a previously healthy 22 year old primi at approximately 20 weeks gestation who presented to us with weakness in all the four limbs since 3 days and vomiting off and on since 6 weeks. This report is unusual but important as it is a rapidly treatable or reversible paresis. Herein this report.

Keywords-Hypokalemic, hyperemesis gravidarum, pregnancy, quadriparesis, reversible.
\end{abstract}

\section{Introduction}

Hypokalemic paralysis is an uncommon entity with varied etiology including renal tubular acidosis and primary hyperaldosteronism. The hypokalemic paralysis is a disease characterized by the development of acute muscular weakness, associated with low levels of blood potassium $(<3.5 \mathrm{meq} / \mathrm{L})$. Weakness typically recovers when potassium level returns to normal. Management plan includes appropriate diagnosis, potassium replacement, potassium sparing diuretics,avoidance of triggers and issues related to operation and pregnancies. Hypokalemic periodic paresis is an autosomal dominant disorder affecting muscles involving the abnormal function of ion channels [1]

\section{Case Report}

We describe a previously healthy 22 year old primi at approximately 20 weeks gestation who presented to us with weakness in all the four limbs since 3 days and vomiting off and on since 6 weeks. She had no difficulty in respiration or swallowing or weakness of face and neck muscles. There was no history of trauma, headache, recent diarrhea, neck-pain, bowel/bladder disturbances and previous such episodes prior to onset of weakness. Her parents and siblings had no history of similar episode or any other significant illness. She also denied use of any injectables prior to hospitalization. On admission she was hemodynamically stable with a pulse rate of 100/minute and a blood pressure of $120 / 80 \mathrm{~mm} \mathrm{Hg}$. She was conscious and oriented to time place and person however power in all the four limbs were grade 2 by MRC(Medical Research Council) scale with diminished deep tendon reflexes in both upper and lower limbs and normal plantar reflexes. Cranial nerve functions were intact and so was the sensory function. Guillain-Barre syndrome was ruled out.

Per abdomen examination revealed uterus of 20-22 week size. Examination of respiratory system and cardiovascular system was unremarkable. Ultrasonography performed was normal. She was administered intravenous fluids and given supportive management keeping in view vomiting which had been troubling her for six weeks. The biochemical profile sent at the time of admission revealed a very low potassium levels of 1.42 $\mathrm{mEq} / \mathrm{L}$. Thyroid profile was normal. Intravenous potassium was replaced which lead to remission of symptoms. Her power improved and she was discharged with potassium supplements and was advised frequent follow ups for fetal and maternal well being.

\section{Discussion-}

Failure to properly diagnose hypokalemic paralysis can be fatal, whereas prompt recognition and rapid replacement of potassium can resolve the symptoms quickly and completely. When possible, the underlying cause must be adequately addressed to prevent the persistence or recurrence of paralysis.

Any pregnancy with paresis is a high risk pregnancy. In this case the patient was a primi with 5 months amenorrhoea and presented with complaints of vomiting since six weeks. Hyperemesis gravidarum (HG) is a severe form of nausea and vomiting in pregnancy different from the more common nausea and vomiting known as morning sickness. The nausea and vomiting usually begins in the first or second month of pregnancy. Fever, abdominal pain, or late onset of nausea and vomiting usually indicate another condition, such as appendicitis, gall bladder disease, gastritis, hepatitis or infection which was ruled out in this case.

Approach to a patient with hypokalemic paralysis should be a careful search for the etiology and potassium replacement therapy. Although the commonest form of hypokalemic paralysis is the hereditary variety, some patients may present with episodic weakness in thyrotoxicosis.[2]Hypokalemic periodic paralysis, may be induced by betamethasone injections during pregnancy and is reversible[3]. Assaying of thyroid 
function in patients with hypokalemic paralysis distinguishes thyrotoxic periodic paralysis from other forms of hypokalemic periodic paralysis.

The main steps in the management include exclusion of other causes of hypokalemia, potassium replacement, hydration and close monitoring of the cardiac rhythm and serum potassium levels. The underlying cause must be searched for to prevent the persistence or recurrence of paralysis. [4] Acetazolamide, dichlorphenamide, or potassium-sparing diuretics decrease attack frequency and severity on chronic basis but not acutely. [5]

\section{Conclusion}

As acute onset nontraumatic quadriparesis in pregnancy is usually reversible and rapidly treatable therefore these patients should be screened for potassium levels which may be deranged secondary to various causes including hyperemesis gravidarum.

\section{References}

[1]. A. Elbaz, J. Vale-Santos, K. Jurkat-Rott, P. Lapie, R. A. Ophoff, B. Bady et al. Hypokalemic Periodic Paralysis and the Dihydropyridine Receptor (CACNLIA3): Genotype/Phenotype Correlations for Predominant Mutations and Evidence for the Absence of a Founder Effect in 16 Caucasian Families. Am J Hum Genet. 1995; 56: 374-380.

[2]. Cesur M, Ilgin SD, Baskal N, Gullu S. Hypokalemic paralysis is not just a hypokalemic paralysis.Eur J Emerg Med. 2008;15:150-3.

[3] Teagarden CM, Picardo CW. Betamethasone-induced hypokalemic periodic paralysis in pregnancy.Obstet Gynecol. 2011;117:433-5.

[4]. Alkaabi JM, Mushtaq A, Al-Maskari FN, Moussa NA, Gariballa S. Hypokalemic periodic paralysis: a case series, review of the literature and update of management.Eur J Emerg Med. $2010 ; 17: 45-7$.

[5] .Jacob O Levitt. Practical aspects in the management of hypokalemic periodic paralysis. J Transl Med. 2008; 6: 18. 